\begin{tabular}{||l|l|}
\hline Citation & $\begin{array}{l}\text { Dhuldeo Kachare, Xiao-Ping Song, Piet Herdewijn (2014), } \\
\text { Phospho-carboxylic anhydride of a homologated nucleoside leads to } \\
\text { primer degradation in the presence of a polymerase } \\
\text { Bioorg. Med. Chem. Lett., 24, 817-820. }\end{array}$ \\
\hline Archived version & This is the Post print version of the manuscript \\
\hline Published version & 10.1016/j.bmcl.2013.12.093 \\
\hline Journal homepage & http://www.journals.elsevier.com/bioorganic-and-medicinal-chemistry-letters/: \\
\hline Author contact & $\begin{array}{l}\text { Piet.Herdewijn@rega.kuleuven.be } \\
+32(0) 16337368\end{array}$ \\
\hline IR & https://lirias.kuleuven.be/handle/123456789/440620 \\
\hline
\end{tabular}

(article begins on next page) 


\title{
Phospho-carboxylic anhydride of a homologated nucleoside leads to primer degradation in the presence of a polymerase.
}

\author{
Dhuldeo Kachare ${ }^{\mathrm{a}}$, Xiao-Ping Songa, Piet Herdewijn ${ }^{\mathrm{a} 1}$
}

${ }^{a}$ Medicinal Chemistry, Rega Institute for Medical Research, KU Leuven, Minderbroedersstraat 10, 3000 Leuven, Belgium

\begin{abstract}
Starting from thymidine, through a series of key synthetic transformations (e.g. Wittig reaction, hydroboration, Mitsunobu reaction and TEMPO oxidation) a nucleoside homologue bearing a phospho-carboxylic anhydride group at 6' position was synthesized. The potential of polymerases to catalyze amide bond formation was investigated by using a modified primer with an amino group at 3' position and the synthesized phosphoanhydro compound as substrate. Unfortunately, we did not observe the desired product either by gel electrophoresis or mass spectrometry. In contrast, the instability of the phosphoanhydro compound could lead to pyrophosphate formation and thus, to pyrophosphorolysis of the primer rather than to primer extension.
\end{abstract}

Keywords: Phospho-carboxyl anhydride; nucleosides; polymerases; phosphorolysis; homologated AZT

\section{Graphical Abstract}<smiles>Cc1cn(C2CCOCC2O)c(=O)[nH]c1=O</smiles><smiles></smiles>

\footnotetext{
${ }^{1}$ Corresponding author: Tel. +32 16 337387; Fax: +32 16 337340; E-mail: Piet.Hedewijn@rega.kuleuven.be
} 
In coeval life, proteins and nucleic acids are intricately dependent upon each other for a host of functions. Protein enzymes (polymerases) are necessary for the synthesis of DNA and RNA, while nucleic acids (ribosomes) are necessary for the synthesis of proteins. According to the RNA world hypothesis, early life used nucleic acids for both information storage and chemical catalysis before the emergence of protein enzymes. However, it still remains unclear how nucleic acids were able to assemble and replicate before the advent of protein enzymes. This means that in contemporary life, proteins can catalyze phosphodiester bond formation (nucleic acid synthesis) and nucleic acids can catalyze amide bond formation (protein synthesis). Our motivation in this context comes from the recently proposed 'Molecular Midwife' hypothesis ${ }^{1}$ and has the aim to investigate if polymerases can also catalyze amide bond formation. Furthermore, investigations on alternative information systems in the field of synthetic biology ask for a careful mapping of the substrate specificity of natural enzymes, such as polymerases. ${ }^{2}$

In a first effort to test the potential of polymerases to catalyze an amide bond, we considered the synthesis of a modified nucleoside with a carboxylate group at 6' position and an amine group at 3' position. The carboxylate group needs to be activated (for example as mixed anhydride with phosphate) before the compound can be tested as substrate for polymerases. The selection of a 3'-amino group is based on a) the possibility to get chain elongation reaction and b) the introduction of a positive charge in the oligonucleotide (after incorporation reaction) to facilitate detection by gel chromatography and mass spectrometry. Due to the expected difficulties in obtaining a carboxylate activated, unprotected amino acid, we decided to test the reaction with a 3'-azido congener. The azido group could then be reduced to an amino group after incorporation, if that would be necessary for analytic purposes.

The synthetic scheme started from commercially available thymidine $\mathbf{1}$, in which compounds $\mathbf{2}$ and $\mathbf{3}$ are synthesized by using literature procedures reported by Tronchet et. al. ${ }^{3}$
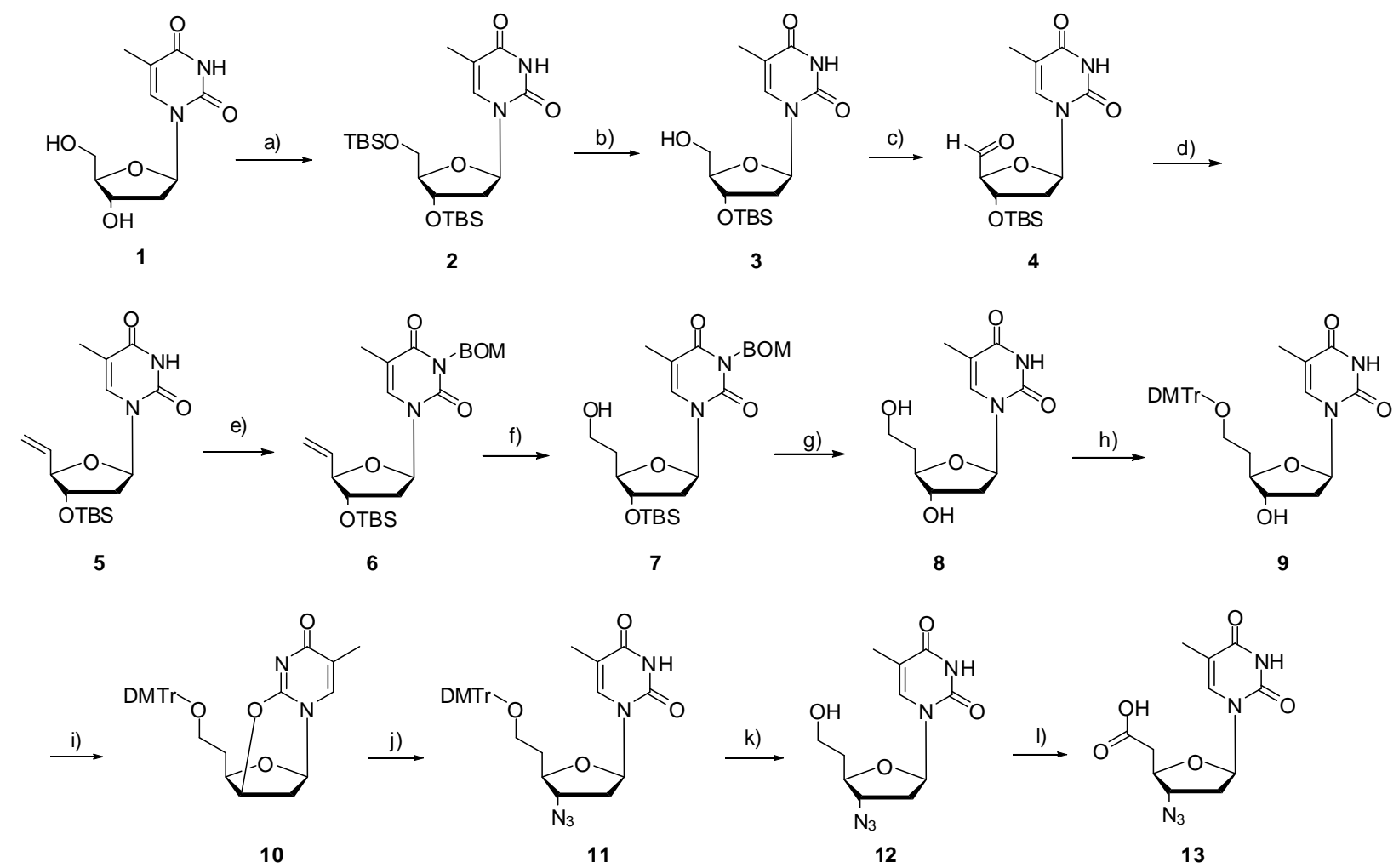

Scheme 1. Reagents and conditions: a) TBDMSCl, Py, added at rt for $1 \mathrm{~h}$, then stirred at $60-70{ }^{\circ} \mathrm{C}, 22 \mathrm{~h}, 98.8 \%$; b) TFA/ $\mathrm{H}_{2} \mathrm{O}$ (10:1, v/v), DCM, rt, 45 min then ice bath for $30 \mathrm{~min}, 80 \%$; c) Dess-Martin periodinane, MeCN, $80{ }^{\circ} \mathrm{C}$; d) 
methyltriphenylphosphonium bromide, potassium tert-butoxide, THF, added at $0{ }^{\circ} \mathrm{C}$ and stirred at rt, overnight, $53 \%$; e) BOM$\mathrm{Cl}, \mathrm{DBU}, \mathrm{MeCN}, 0^{\circ} \mathrm{C}$ to rt, 2h, $80.6 \%$; f) 9-BBN, THF, added at $0{ }^{\circ} \mathrm{C}$ and stirred at rt, overnight; then added $\mathrm{NaOH}$ and $\mathrm{H}_{2} \mathrm{O}_{2}$ at $0{ }^{\circ} \mathrm{C}$ and stirred at rt, 6 h, $57.2 \%$; g) Pd/C $10 \%$ wt., cyclohexene, MeOH, reflux, 57 \%; h) DMTr-Cl, pyridine, rt, 70.7 \%; i) $\mathrm{PPh}_{3}$, DIAD, MeCN, $0{ }^{\circ} \mathrm{C}, 12 \mathrm{~h}, 87.7 \%$; j) NaN , DMF, $140{ }^{\circ} \mathrm{C}, 12$ h, $85.3 \%$; k) 3 \% trichloroacetic acid, DCM, rt, 66.7 \%; l) TEMPO, bis(acetoxy)iodobenzene, MeCN: $\mathrm{H}_{2} \mathrm{O}(1: 1, \mathrm{v} / \mathrm{v})$, rt, overnight, $57.7 \%$.

Compound 3 was oxidized to $\mathbf{4}$ using Dess-Martin periodinane (DMP) reagent. Wittig reaction was carried out on $\mathbf{4}$, using potassium tert-butoxide and methyl triphenylphosphonium bromide in THF to afford the vinyl compound 5. The vinyl compound 5 was reacted with benzylchloromethyl ether (BOM-Cl) to protect the $\mathrm{NH}$ group in thymine ring to furnish compound 6. BOM protected compound 6 was reacted with 9-borabicyclo[3.3.1]nonane (9-BBN) and subsequent treatment with hydrogen peroxide in alkaline medium to give $7 .{ }^{4}$ The removal of BOM group by treatment with Pd/C $10 \%$ wt. in MeOH in presence of cyclohexene under reflux conditions, also lead to removal of the TBS group at the 3' position affording 8. Homologated thymidine 8 was protected at the 6'-O-position with 4, 4'-dimethoxytrityl chloride in pyridine yielding compound 9. The subsequent synthesis of compound $\mathbf{1 1}$ involved an inversion of the 3'-carbon atoms from S-configuration to Rconfiguration which was accomplished in two steps. Cyclisation of compound $\mathbf{9}$ under Mitsunobu condition gave the $\mathrm{O}^{2}$, 3'anhydro derivative 10, which is a useful intermediate for substitutions at the 3'-position. Opening of the aza-enol-ether in $\mathbf{1 0}$ with sodium azide in DMF gave protected AZT derivative 11. ${ }^{5}$ Compound 11 was deprotected with $3 \%$ trichloroacetic acid to furnish homologated alcohol 12, which was subsequently oxidized at 6' position to afford the corresponding carboxylic acid 13 (Scheme 1).

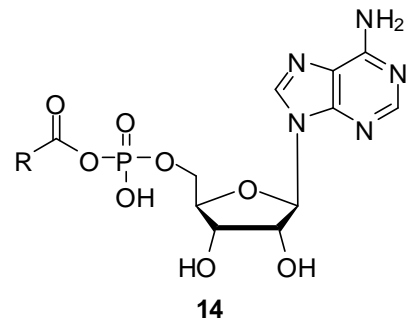

Acyl-AMP

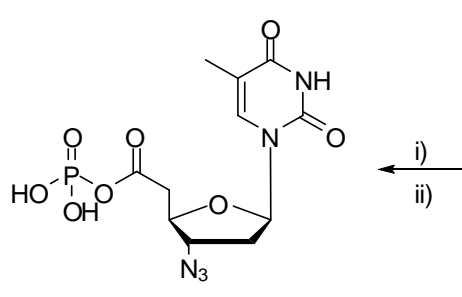

15

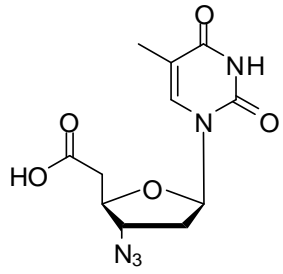

13

Mixed anhydride

Scheme 2. Chemical structure of acyl phosphate (14) and mixed anhydride (15); (i) ethylchloroformate, n-Bu3 N, 1,4-dioxane, $0{ }^{\circ} \mathrm{C}$-rt; (ii) $0.5 \mathrm{M}$ tri-n-butylammonium phosphate, DMF.

Acyl monophosphates (14) are known as intermediates in biosynthesis, but unfortunately due to the low stability their synthesis and purification are not much explored. As far as we know, there is no literature dealing with the synthesis of mixed anhydrides (15) in which the nucleoside moiety contains a carboxylic acid functionality (Scheme 2).

Our first attempt to obtain anhydride 15 from compound 13 by using bis(trimethylsilyl)-tributylstannyl phosphate was unsuccessful. ${ }^{6}$ Further efforts for its synthesis by using diethyl chlorophosphate, diphenyl chlorophosphonate, and bis(tetraethyl ammonium) ethyl phosphate were also unsuccessful. ${ }^{7,8}$ Therefore we focused on a two-step reaction. The acid 13 was first reacted with ethylchloroformate and tri-n-butylamine to furnish the corresponding anhydride, then the obtained intermediate was reacted with tri-n-butylammonium phosphate (0.5 M in DMF) affording the desired mixed anhydride 15 (Scheme 2). ${ }^{9,} 10$ The tri-n-butylammonium phosphate (0.5 M in DMF) reagent was synthesized by using a reported procedure by El-Tayeb et al. ${ }^{11}$ Compound 15 , however, was unstable and was isolated by partitioning the reaction mixture between ethyl acetate and cold water. The product was collected in the aqueous layer and isolated by freeze drying. Further purification of compound $\mathbf{1 5}$ was carried out by HPLC with C18 column using a gradient elution buffer of triethylammonium acetate (TEAA) and acetonitrile. 
In the context of probing template-directed amide bond formation by polymerases, compound $\mathbf{1 5}$ bearing a phospho-carboxylic acid anhydride function at 6'-position of sugar moiety was used as substrate, a modified primer (P1) was installed with 3'$\mathrm{NH}_{2}-2$ ', 3'-dideoxycytidine at 3' terminal and template T1 was employed as primer-template duplex. Initially, the substrate properties of compound 15 were evaluated by four enzymes (HIV-1 RT, Therminator, Taq and VentR ${ }^{\circledR}$ (exo-) DNA polymerase) from different families. Unfortunately, using HIV-1 RT, Taq, and VentR ${ }^{\circledR}$ (exo-) DNA polymerase as catalyst, no primer extension was obtained. A small amount of primer extension $(\mathrm{P}+1, \mathrm{P}+2)$ was observed using Therminator ${ }^{\mathrm{TM}}$ DNA polymerase as catalyst, and also several bands were detected below the primer baseline (Figure 1A).

Table 1. Overview of primer-template complexes used in the enzymatic polymerization

\begin{tabular}{|l|l|}
\hline Primer 1 (P1) & 5'-CAGGAAACAGCTATGACNH 2 -3' \\
\hline Primer 2 (P2) & 5'-CAGGAAACAGCTATGAC-3' \\
\hline Template 1 (T1) & 3'-GTCCTTTGTCGATACTGACTGC-5' \\
\hline
\end{tabular}

Gel electrophoresis is a popular tool used to separate nucleic acids based on both their molecular weight and charges. In our case, when compound 15 would be successfully incorporated into the primer (P1), the extended oligonucleotide will keep the same number of negative charges and an increase in molecular weight of $277 \mathrm{~m} / \mathrm{z}$. Regarding the minor difference between primer P1 and the formed product, protonation of the amine group of P1 might be a feasible way to change the gel mobility difference of $\mathrm{P} 1$ and $[\mathrm{P} 1+\mathbf{1 3}]$. Analytical gels of the Therminator ${ }^{\mathrm{TM}}$ DNA polymerase catalyzed reaction mixture at two different $\mathrm{pH}$ values (gel $1 \mathrm{pH}=6.25$, gel $2 \mathrm{pH}=8.3$ ) were performed in order to detect the small difference between P1 and the primer extended product. However, no significant difference was detected for the gel picture obtained at lower $\mathrm{pH}$ value comparing to the gel showed in Figure $1 \mathrm{~A}(\mathrm{pH}=8.3)$. 


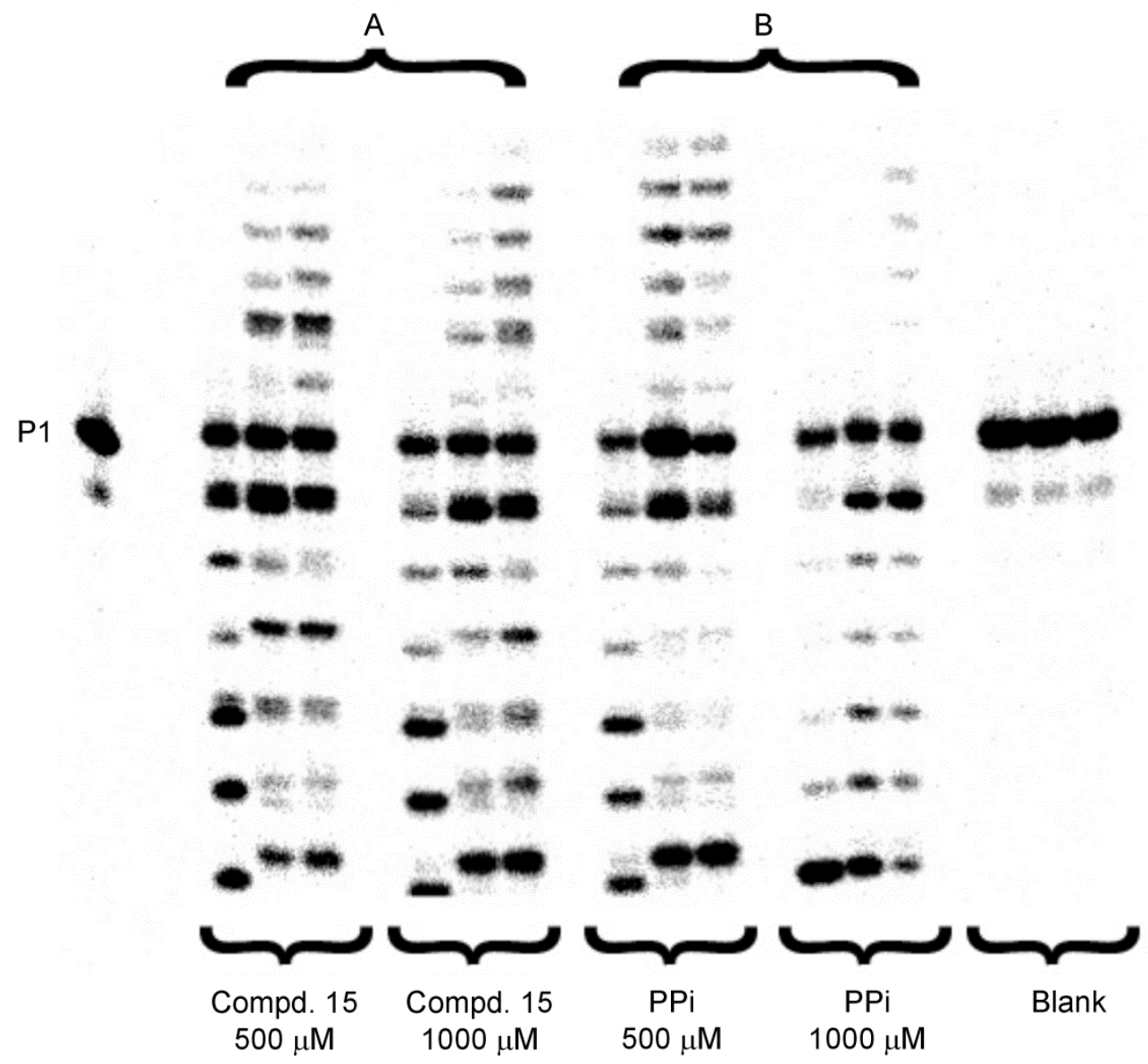

Figure 1. A: incorporation study of compound $\mathbf{1 5}$ as substrate; B: enzymatic reaction using pyrophosphate (PPi) as substrate; Blank: no substrate in the reaction. [P1T1] $=125 \mu \mathrm{M}$; [Therminator ${ }^{\mathrm{TM}} \mathrm{DNA}$ pol] $=0.05 \mathrm{U} / \mu \mathrm{L}$; time points: 10, 30 and $90 \mathrm{~min}$. Furthermore, investigation to detect the incorporated product was performed by mass spectrometry analysis. The sample was prepared with unlabeled primer P1 and template T1. After reaction the abundant salts in the reaction were removed by Illustra ${ }^{\mathrm{TM}}$ Microspin $^{\mathrm{TM}}$ G-25 column. Unfortunately, no desired product was detected.

Enzymatic DNA polymerization is a reversible reaction. ${ }^{12}$ The presence of high concentration of pyrophosphate in the reaction mixture can lead to pyrophosphorolysis of primer or template to form nucleoside triphosphates. ${ }^{12}$ Interestingly, as shown in Figure 1, similar patterns were obtained for the reaction when using either compound $\mathbf{1 5}$ (Figure 1A) or pyrophosphate (Figure 1B) as substrate. The above observation demonstrates that the presence of compound $\mathbf{1 5}$ can lead to pyrophosphorolysis of primer and/or template in the presence of Therminator DNA polymerase. Most probably, pyrophosphate is formed in the reaction mixture by reaction of organic phosphate with $\mathbf{1 5}$. The necessary phosphate could be originated in the partial degradation of compound $\mathbf{1 5}$ in the reaction medium, which is an unstable compound. No primer degradation or extension was observed when the reaction was performed without enzyme (picture not shown). Primer degradation was significantly suppressed in the presence of pyrophosphatase (PPase) (Figure 2A). Additionally, when using the unmodified oligonucleotide as primer (P2) in the Therminator ${ }^{\mathrm{TM}}$ DNA polymerase catalyzed reaction, compound $\mathbf{1 5}$ can cause similar primer degradation pattern as showed in Figure 2B. The above experiments show that compound $\mathbf{1 5}$ could induce similar primer degradation as pyrophosphate in the presence of Therminator ${ }^{\mathrm{TM}}$ DNA polymerase, due to pyrophosphate generation in the reaction mixture. The observed primer extension (Figure 2) might be explained by the incorporation of dNMP's (natural deoxynucleoside 
monophosphate), which were formed during degradation (as nucleoside triphosphates) of primer and/or template by pyrophosphorolysis in the presence of Therminator ${ }^{\mathrm{TM}}$ DNA polymerase. ${ }^{2}$

A
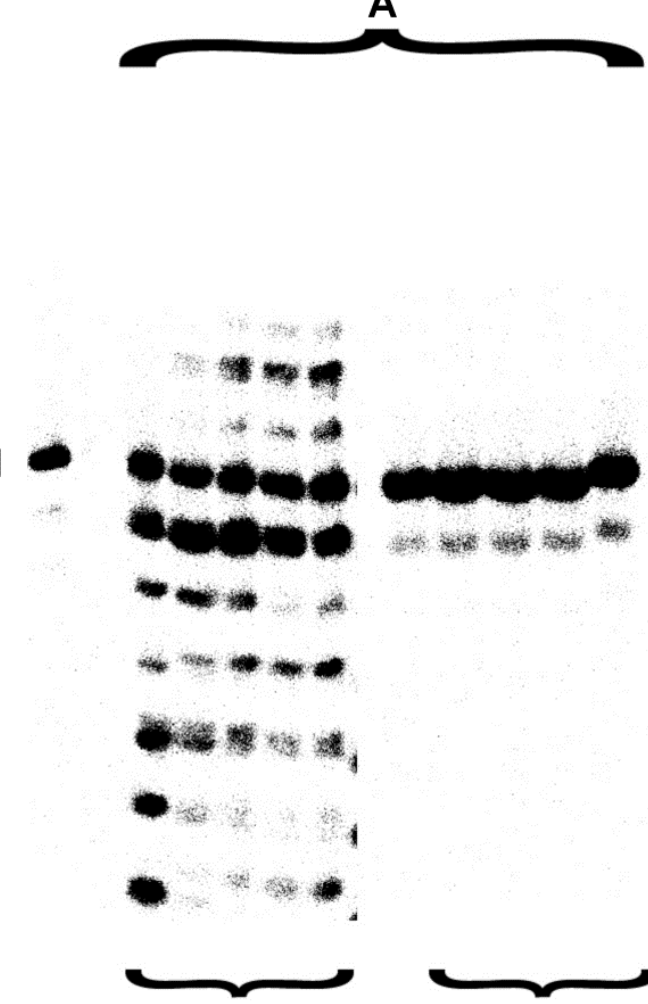

Compd. 15

$500 \mu \mathrm{M}$
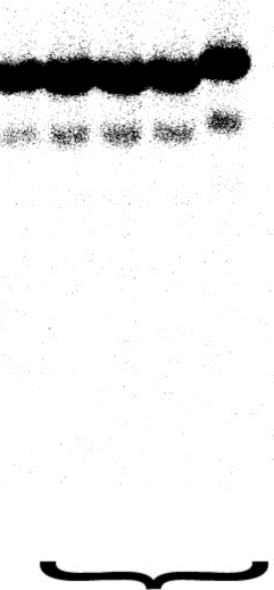

Compd. 15

$+$

PPase

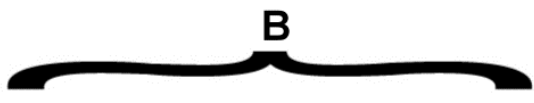

P2
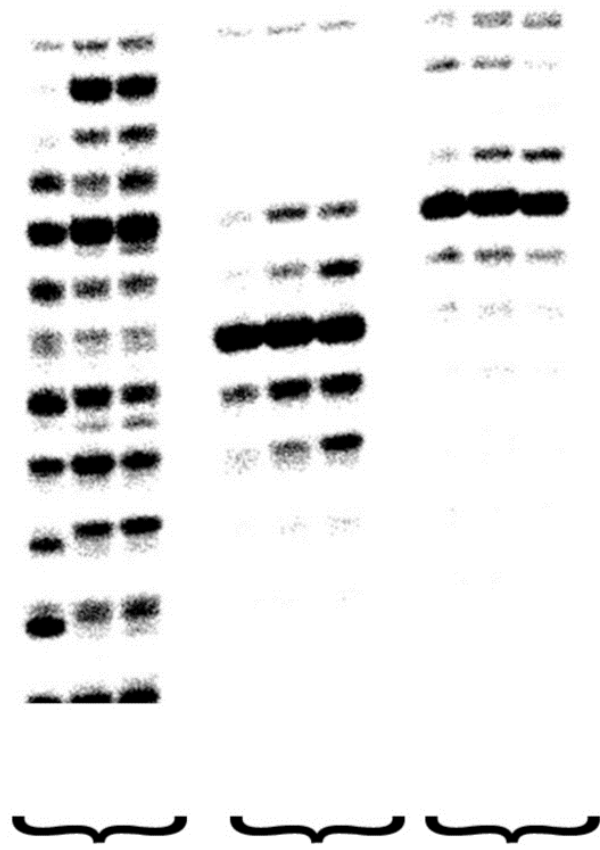

$\begin{array}{cc}\text { Compd. } 15 & \text { Comp. } 15 \\ 500 \mu \mathrm{M} & + \\ & \text { PPase }\end{array}$

Figure 2. A: incorporation study of compound 15 using P1T1 as primer/template duplex in absence and presence of pyrophosphates, time points: 10, 20, 30 60, $120 \mathrm{~min}$; B: incorporation study of compound 15, in absence and presence of pyrophosphatase using P2T1 as primer/template duplex, time points: 10, 30 and $90 \mathrm{~min}$. [P1T1] = $125 \mu \mathrm{M},[\mathrm{P} 2 \mathrm{~T} 1]=125 \mu \mathrm{M}$, [Therminator ${ }^{\mathrm{TM}} \mathrm{DNA}$ pol] $=0.05 \mathrm{U} / \mu \mathrm{L}$, [PPase $]=0.01 \mathrm{U} / \mu \mathrm{L}$, [compound 15] $=500 \mu \mathrm{M},[\mathrm{dTTP}]=50 \mu \mathrm{M}$.

The synthesis of the 5'- one carbon extended nucleoside homologue was readily accomplished from commercially available thymidine. The key steps in the synthesis were the Wittig reaction and the hydroboration of the thymidine precursor with 9BBN to furnish the corresponding homologated alcohol 12. The inversion of configuration and introduction of the azide group at 3' position was achieved by Mitsunobu reaction. Oxidation of compound 12 using TEMPO leads to the corresponding acid 13. Finally, a two-step reaction was explored in order to obtain the mixed anhydride $\mathbf{1 5}$. Compound $\mathbf{1 3}$ was first activated by ethylchloroformate, then the activated nucleoside carboxylic acid was reacted with tri-n-butylammonium phosphate (0.5 M in DMF) to give the desired product.

The potential of polymerases to catalyze the amide bond formation between the 3'-amino group of the modified primer and the 5'-carboxylate group of compound $\mathbf{1 5}$ on a template directed manner was investigated by a series of enzymes from different families. Unfortunately, no product formation was detected either by mass spectrometry or gel electrophoresis. One possible explanation could be that compound $\mathbf{1 5}$ has not the appropriate geometry to be recognized by the active site of polymerases. On the other hand, the instability of the phospho-anhydride group of the mixed anhydride $\mathbf{1 5}$ may lead to the formation of a 
significant amount of pyrophosphate and, thus, rather to primer degradation by enzymatic pyrophosphorolyis than to primer extension.

\section{Acknowledgements}

The research leading to these results has received funding from the European Research Council under the European Union's Seventh Framework Program (FP7/2007-2013)/ERC Grant agreement n8 ERC-2012-ADG_20120216/320683. XNA=xenobiotic nucleic acids. Dr. D. Kachare received a research grant from Federaal Wetenschapsbeleid. Dr. Xiao-Ping Song a research associate of the Belgian National Fund for Scientific Research (FWO).

\section{References:}

1. $\quad$ Horowitz, E. D.; Engelhart, A. E.; Chen, M. C.; Quarles, K. A.; Smith, M. W.; Lynn, D. G.; Hud, N. V. Proc. Natl. Acad. Sci. U. S. A. 2010, 107, 5288

2. Jang, M.-Y.; Song, X.-P.; Froeyen, M.; Marlière, P.; Lescrinier, E.; Rozenski, J.; Herdewijn, P. Chem. Biol. 2013, 20, 416.

3. Tronchet, J. M.; Kovacs, I.; Dilda, P.; Seman, M.; Andrei, G.; Snoeck, R.; De Clercq, E.; Balzarini, J. Nucleos. Nucleot. Nucleic Acids 2001, 20, 1927.

4. $\quad$ von Matt, P.; Altmann, K. H. Bioorg. Med. Chem. Lett. 1997, 7, 1553.

5. $\quad$ Ludek, O. R.; Balzarini, J.; Meier, C. Eur. J. Org. Chem. 2006, 932.

6. $\quad$ Yamaguchi, K.; Kamimura, T.; Hata, T. J. Am. Chem. Soc. 1980, 102, 4534.

7. $\quad$ Procopiou, P. A.; Biggadike, K.; English, A. F.; Farrell, R. M.; Hagger, G. N.; Hancock, A. P.; Haase, M. V.; Irving, W. R.; Sareen, M.; Snowden, M. A.; Solanke, Y. E.; Tralau-Stewart, C. J.; Walton, S. E.; Wood, J. A. J. Med. Chem. 2001, 44, 602.

8. $\quad$ Kluger, R.; Li, X. F.; Loo, R. W. Can. J. Chem. 1996, 74, 2395.

9. $\quad$ Kreimeyer, A.; UghettoMonfrin, J.; Namane, A.; HuynhDinh, T. Tetrahedron Lett. 1996, 37, 8739.

10. Bonnaffe, D.; Dupraz, B.; Ughettomonfrin, J.; Namane, A.; Dinh, T. H. Tetrahedron Lett. 1995, 36, 531.

11. $\quad$ El-Tayeb, A.; Qi, A. D.; Muller, C. E. J. Med. Chem. 2006, 49, 7076.

12. Deutscher, M. P.; Kornberg, A. J. Biol. Chem. 1969, 244, 3019. 

